\title{
The Pseudovariety of Semigroups of Triangular Matrices over a Finite Field
}

\author{
J. Almeida \\ S. W. Margolis \\ M. V. Volkov* \\ Dedicated to Imre Simon on the occasion of his 60th birthday
}

\begin{abstract}
We show that semigroups representable by triangular matrices over a fixed finite field form a decidable pseudovariety and provide a finite pseudoidentity basis for it.
\end{abstract}

\section{Background and motivation}

The main results of this paper were motivated by one of the fundamental theorems of Imre Simon, namely, by his elegant algebraic characterization of the class of piecewise testable languages [21, 22]. This celebrated theorem was one of the main illuminating examples for the creation of the theory of pseudovarieties of finite semigroups and varieties of recognizable languages. By now there are a number of proofs $[1,11,12,23,26]$ based on different approaches whose sources range from fairly concrete calculations in finite transformation semigroups to highly abstract constructions of model theory or profinite topology and so it has become a crossing where various profound ideas and techniques meet. Thus Simon's Theorem has motivated a generation of researchers who have studied the relationship between finite semigroup theory and theoretical computer science.

There are highly non-trivial purely algebraic consequences of Simon's Theorem. Straubing [24] proved that a finite semigroup is $\mathscr{J}$-trivial if and only if, for some $n$, it divides the semigroup $U_{n}$ of all $n \times n$ upper triangular Boolean matrices with all 1's on the main diagonal. While it is easy to check that the semigroup $U_{n}$ is $\mathscr{J}$-trivial, it is very difficult to prove that conversely every finite $\mathscr{J}$-trivial semigroup divides $U_{n}$ for some $n$. The original proof in [24]

\footnotetext{
*This work was completed in December 2003 when the third named author was visiting the University of Porto with the support of F.C.T. through Centro de Matemática and the project POCTI/32817/MAT/2000. He was also supported by the Science and Education Ministry of Russian Federation, grants E02-1.0-143 and 2227.2003.01. The first named author also acknowledges support of F.C.T. through Centro de Matemática and the project POCTI/32817/MAT/2000, which is funded, in part, by FEDER. The second named author was supported by the Excellency Center "Group Theoretic Methods in the Study of Algebraic Varieties" of the Israeli Science Foundation and by the Binational Science Foundation of the USA and Israel, grant 1999298/1. All three authors acknowledge the support from INTAS through the Network project 99-1224.
} 
uses Simon's theorem in a crucial way, and no straightforward algebraic proof of this simple to state result is known so far.

A direct linear analogue of the semigroup $U_{n}$ is the group $U_{n}^{*}(K)$ of all $n \times n$ upper triangular matrices over a field $K$ with all 1's on the main diagonal. Such groups have been studied intensely for more than 100 years. If $K$ is a finite field of characteristic $p$, then an elementary counting argument shows that in fact $U_{n}^{*}(K)$ is a $p$-group. It is a standard and classical fact that conversely every finite $p$-group is isomorphic to a group of upper triangular matrices (of some size) with all 1's on the main diagonal over any finite field of characteristic $p$ (see, e.g., [9, Theorem 3.1.2]).

Thus we see algebraically, that there is a deep connection between finite $\mathscr{J}$-trivial semigroups and finite $p$-groups. This analogy also shows up on the language theoretic side. Whereas Simon's theorem tells us that the languages recognized by finite $\mathscr{J}$-trivial monoids are built from looking at the appearance or non-appearance of subwords in a word, that is by doing counting over the two element Boolean semiring, the languages recognized by finite $p$-groups correspond to counting subwords modulo $p$. See [7, Sections VIII.9 and VIII.10] and also [28] for a precise formulation of these results.

Pin and Straubing [18] proved that the monoid pseudovariety generated by the monoid of all upper triangular Boolean matrices also has a natural language theoretic interpretation. Namely, the corresponding variety of languages is precisely the languages of level 2 in the Straubing-Thérien hierarchy [25, 27]. (Recall that the Straubing-Thérien hierarchy is the monoid counterpart of the dot-depth hierarchy by Brzozowski [6]. See [17, Section V.2] for a discussion of the two hierarchies and their relationship.) The monoids of level 1 in the Straubing-Thérien hierarchy are precisely the $\mathscr{J}$-trivial monoids, but as of today, the membership problem for the pseudovariety of Straubing-Thérien level 2 remains one of the most important open problems in finite semigroup theory.

This brings us to the subject of the present paper. We ask here what is the mod- $p$ analogue of the monoid of upper triangular Boolean matrices. Clearly we need to look at the monoid of upper triangular matrices over a finite field of characteristic $p$. Thus we are asking about what finite semigroups can be represented as semigroups of triangular matrices and we enter the world of the classical problem of triangulating a set of matrices over a field. Indeed, the study of simultaneous triangularizability of families of matrices has been considered as an important issue since the mid-19th century, and one can see that many classical branches of algebra such as group, associative ring or Lie algebra theories have offered their specific triangularizability conditions. Rather than trying to survey this vast area, we refer the reader to the recent comprehensive monograph [19]. Not long ago Okniński [16, Section 4.4] found a deep triangularizability criterion for semigroups of matrices. We show that being restricted to the realm of finite semigroups Okniński's criterion admits a fairly natural expression in terms of pseudovariety theory. In particular, we prove that finite semigroups triangularizable over a fixed finite field form a pseudovariety with decidable membership, and moreover, provide a finite pseudoidentity basis for this pseudovariety. These results imply several interesting corollaries of both 
an algebraic and a geometric nature. The three authors along with Benjamin Steinberg are preparing a separate paper devoted to further development of these results and their applications to language theory and to the representation theory of finite semigroups.

The paper is structured as follows. In Section 1 we recall some basics of finite semigroup theory and then formulate and discuss our main results. Section 2 collects a few properties of semigroups of triangular matrices and presents an adaptation of Okniński's triangularizability criterion for the case of finite semigroups. Section 3 provides the necessary preliminaries on groups. In Section 4 we prove the main results, and Section 5 contains some interesting examples.

\section{Main results}

We assume the reader's acquaintance with standard concepts of semigroup theory (in particular, with the definition of Green's relations). They all can be found in the books $[5,14]$.

Recall that a semigroup pseudovariety is a class of finite semigroups closed under forming finitary direct products and taking homomorphic images and subsemigroups. The following well-known pseudovarieties play a distinguished role in this paper: the pseudovariety DS of all finite semigroups whose regular $\mathscr{D}$ classes are subsemigroups and the pseudovariety $\mathrm{G}_{p}$ of all finite $p$-groups where $p$ is a prime number. We also make use of an operator on pseudovarieties. For each pseudovariety $\mathrm{H}$ of finite groups, $\overline{\mathrm{H}}$ denotes the class of all finite semigroups whose subgroups belong to $\mathrm{H}$. It is known (cf. [7, Proposition B.V.10.4]) and easy to see that $\overline{\mathrm{H}}$ is a pseudovariety.

Now we fix an arbitrary finite field $\mathbb{F}$ and denote its characteristic by $p$ and its order by $q$ (so $q$ is a power of $p$ ). By $T_{n}(\mathbb{F})$ we denote the semigroup of all upper triangular $n \times n$ matrices over $\mathbb{F}$. A finite semigroup $S$ is said to be triangularizable over $\mathbb{F}$ if $S$ embeds into $T_{n}(\mathbb{F})$ for some $n$. Further, by $U T_{n}(\mathbb{F})$ we denote the subsemigroup of $T_{n}(\mathbb{F})$ consisting of all unitriangular matrices (that is, matrices whose main diagonal entries are equal to either 0 or 1). A finite semigroup that embeds into $U T_{n}(\mathbb{F})$ for some $n$ is said to be unitriangularizable over $\mathbb{F}$.

Our first result is the following.

Proposition 1.1 The class $\mathrm{UT}_{p}$ of all finite semigroups unitriangularizable over a given finite field of characteristic $p$ is the pseudovariety $\overline{\mathrm{G}_{p}} \cap \mathrm{DS}$.

Even though Proposition 1.1 is not the main result of the paper, let us discuss it in detail because some of our comments also apply to more involved statements below. First of all, observe that the pseudovariety $\mathrm{UT}_{p}$ depends only on the characteristic of the ground field and does not depend on its order. Moreover, the fact that pseudovarieties come into play is somewhat unexpected. While it is trivial that the class $\mathrm{UT}_{p}$ is closed under taking subsemigroups and forming finitary direct products, it is far from being obvious that every homomorphic image of a unitriangularizable semigroup is again unitriangularizable. Finally, observe that given a finite semigroup $S$ (by its Cayley table, say), one 
can efficiently check if the subgroups of $S$ are $p$-groups and the regular $\mathscr{D}$-classes of $S$ are subsemigroups. (Here the word 'efficiently' means that the tests can be performed in time bounded by a polynomial of the size of $S$.) In other words, we may conclude that the property of being unitriangularizable over a fixed finite field is decidable in polynomial time.

Now we turn to the general case. In order to formulate a description of triangularizable semigroups, let $\mathrm{G}_{p} \mathrm{Ab}^{q-1}$ stand for the collection of all finite groups $G$ such that $G$ is an extension of a $p$-group by an Abelian group of exponent dividing $q-1$. It is easy to verify that $\mathrm{G}_{p} \mathrm{Ab}^{q-1}$ is a pseudovariety. We denote by $\mathcal{E} \bar{G}_{p}$ the class of all finite semigroups whose idempotent-generated subsemigroups belong to $\overline{\mathrm{G}_{p}}$. Again, $\mathcal{E} \overline{\mathrm{G}_{p}}$ is easily seen to be a pseudovariety.

THEOREM 1.2 The class $\mathrm{T}_{p}^{q}$ of all finite semigroups triangularizable over the field of characteristic $p$ and order $q$ is the pseudovariety $\overline{\mathrm{G}_{p} \mathrm{Ab}^{q-1}} \cap \mathrm{DS} \cap \mathcal{E} \overline{\mathrm{G}_{p}}$.

Here, in contrast to Proposition 1.1, the answer depends on the order of the ground field. However, it is easy to deduce from Theorem 1.2 a similar description of finite semigroups triangularizable over some finite field of characteristic $p$. Let $\mathrm{G}_{p} \mathrm{Ab}$ denote the pseudovariety of all extensions of a finite $p$-group by a finite Abelian group. Then we have

COROLlary 1.3 The class $\mathrm{T}_{p}$ of all finite semigroups triangularizable over a finite field of characteristic $p$ is the pseudovariety $\overline{\mathrm{G}_{p} \mathrm{Ab}} \cap \mathrm{DS} \cap \mathcal{E} \overline{\mathrm{G}_{p}}$.

Given the Cayley table of a finite semigroup $S$, one can construct the Cayley table of the subsemigroup generated by the idempotents of $S$ in $O\left(|S|^{3}\right)$ time. Therefore Theorem 1.2 and Corollary 1.3 ensure that the properties of being triangularizable over a fixed finite field or over some finite field of a fixed characteristic are decidable in polynomial time.

Of course, using Corollary 1.3 one can easily decide if a given finite semigroup is triangularizable over some finite field of some characteristic. However, the class of all such finite semigroups is not a pseudovariety (it is not closed under finitary direct products).

Next we present a syntactic description of pseudovarieties of triangularizable semigroups. Recall that there are two different ways to characterize semigroup pseudovarieties syntactically, that is, by means of certain equations. The historically first approach, suggested by Eilenberg and Schützenberger [8], deals with sequences of usual identities. Nowadays, however, another way for a syntactic description of pseudovarieties seems to prevail, namely, the approach which is due to Reiterman [20] and which is based on an extension of the notion of an identity to that of a pseudoidentity, that is, an equality between two elements of the free profinite semigroup rather than of the usual free semigroup. The reader is referred to the book [2] for a general theory of pseudoidentities. In the present paper, however, we only deal with very special pseudoidentities, and the meaning of these pseudoidentities can be explained in fairly elementary terms as follows.

Our pseudoidentities are formal equalities between two ' $\omega$-words' built up from the variables $x$ and $y$ by means of the concatenation and raising to 'profinite powers' containing a special symbol $\omega$ in their exponents. For instance, 
$x^{p^{\omega}},(x y)^{\omega} x$ or $x^{\omega-1} y^{\omega-1} x y$ are typical $\omega$-words that we repeatedly use below. If we want to calculate the value of an $\omega$-word under a certain evaluation of its variables in a semigroup $S$ with $\ell$ elements we merely convert it into a usual word by substituting the symbol $\omega$ wherever it occurs by the number $\ell$ ! and then we evaluate the resulting word in $S$. (Thus, the three typical $\omega$-words above become $x^{p^{\ell !}},(x y)^{\ell !} x$ and $x^{\ell !-1} y^{\ell !-1} x y$ if one needs evaluating them in a semigroup with $\ell$ elements.) A finite semigroup $S$ satisfies the pseudoidentity $\pi(x, y)=\rho(x, y)$ where both $\pi(x, y)$ and $\rho(x, y)$ are $\omega$-words if the values of $\pi(x, y)$ and $\rho(x, y)$ coincide under every evaluation of $x$ and $y$ in $S$. We say that a pseudovariety $\mathrm{P}$ is defined by a system $\Sigma$ of pseudoidentities if $\mathrm{P}$ is precisely the class of all finite semigroups satisfying each pseudoidentity in $\Sigma$ (also $\Sigma$ is said to be a pseudoidentity basis for P). For instance, it is well known (cf. [2, Section 8.1]) that the pseudovariety DS is defined by the pseudoidentity

$$
\left((x y)^{\omega}(y x)^{\omega}(x y)^{\omega}\right)^{\omega}=(x y)^{\omega} .
$$

As yet another example, we mention that the pseudovariety $\mathrm{G}_{p}$ can be defined by the pseudoidentities

$$
x^{p^{\omega}} y=y x^{p^{\omega}}=y .
$$

Of course, the pseudoidentities (1.2) hold in a semigroup $S$ if and only if $S$ contains an identity element that is the value of the $\omega$-word $x^{p^{\omega}}$ under each of its evaluations in $S$. It is a common convention to express this fact in the form of the single pseudoidentity $x^{p^{\omega}}=1$; we will always use such shorthand notation when defining pseudovarieties of groups.

We want to stress that the above simplified treatment of pseudoidentities involving $\omega$-words is nevertheless rigorous and completely consistent with the general theory of pseudoidentities. The reader is referred to [3] and [4, Section 2] for various formal approaches to the concept of a profinite power.

Now we return to our problem of a syntactic description of pseudovarieties of triangularizable semigroups. It is known (see [4, Section 2]) and easy to verify that the pseudovariety $\overline{\mathrm{G}_{p}}$ is defined by the pseudoidentity

$$
x^{\omega}=x^{\omega+p^{\omega}} .
$$

Therefore Proposition 1.1 immediately implies

COROLlary 1.4 The pseudovariety $\mathrm{UT}_{p}$ of all finite semigroups that are unitriangularizable over a finite field of characteristic $p$ is defined by the pseudoidentities (1.1) and (1.3).

The situation is much more complicated for the pseudovarieties $\mathrm{T}_{p}^{q}$ and $\mathrm{T}_{p}$. Recall that by Theorem 1.2 and Corollary 1.3, we have $\mathrm{T}_{p}^{q}=\overline{\mathrm{G}_{p} \mathrm{Ab}^{q-1}} \cap \mathrm{DS} \cap \mathcal{E} \overline{\mathrm{G}_{p}}$ and $\mathrm{T}_{p}=\overline{\mathrm{G}_{p} \mathrm{Ab}} \cap \mathrm{DS} \cap \mathcal{E} \overline{\mathrm{G}}_{p}$. An equational description of the pseudovarieties $\overline{\mathrm{G}_{p} A b^{q-1}}$ and $\overline{\mathrm{G}_{p} \mathrm{Ab}}$ makes no real problem in view of the results of [4, Section 3]. There it is shown how to transform any pseudoidentity system defining a given group pseudovariety $\mathrm{H}$ within the class of all finite groups into a pseudoidentity systems defining the pseudovariety $\overline{\mathrm{H}}$ within the class of all finite semigroups so that the two systems contain the same number of pseudoidentities and involve 
the same variables. It is easy to see that the pseudovariety $G_{p} A b^{q-1}$ is defined within the class of all finite groups by the pseudoidentities

$$
\begin{gathered}
\left(x^{q-1} y^{q-1}\right)^{p^{\omega}}=1, \\
\left(x^{\omega-1} y^{\omega-1} x y\right)^{p^{\omega}}=1
\end{gathered}
$$

while pseudovariety $G_{p} A b$ is defined by (1.5) and the following pseudoidentity

$$
\left(x^{p^{\omega}-1} y^{p^{\omega}-1}\right)^{p^{\omega}}=1 .
$$

(For the sake of completeness we provide a proof of these claims in Section 3.) Thus, each of the pseudovarieties $\overline{\mathrm{G}_{p} A b^{q-1}}$ and $\overline{\mathrm{G}_{p} \mathrm{Ab}}$ can be defined by just two pseudoidentities in two variables.

Since we know a single pseudoidentity in two variables that defines the pseudovariety DS, our task seems to reduce to finding a syntactic description of the pseudovariety $\mathcal{E} \overline{\mathrm{G}_{p}}$. However here we encounter a serious problem because the latter pseudovariety cannot be defined by a finite number of pseudoidentities, and moreover, by any system of pseudoidentities involving only finitely many variables. This immediately follows from the main result of [29].

We thus see that the structural descriptions of the pseudovarieties $\mathrm{T}_{p}^{q}$ and $\mathrm{T}_{p}$ provided by Theorem 1.2 and Corollary 1.3 do not translate well into syntactic descriptions. In spite of this fact, we have found a single pseudoidentity in two variables that defines the pseudovarieties $T_{p}^{q}$ and $T_{p}$ within the pseudovarieties $\overline{\mathrm{G}_{p} A b^{q-1}} \cap \mathrm{DS}$ and respectively $\overline{\mathrm{G}_{p} \mathrm{Ab}} \cap \mathrm{DS}$. This is the pseudoidentity

$$
\left(\left((x y)^{\omega} x\right)^{p^{\omega}-1}\left(y(x y)^{\omega}\right)^{p^{\omega}-1}\right)^{p^{\omega}+1}=\left((x y)^{\omega} x\right)^{p^{\omega}-1}\left(y(x y)^{\omega}\right)^{p^{\omega}-1} .
$$

At first glance, the pseudoidentity looks complicated and perhaps somewhat mysterious but we show in Section 4 that it reflects the very core of Okniński's triangularizability criterion. In order to make it and other pseudoidentities look more concise we let $e$ denote the expression $(x y)^{\omega}$. With this convention we may formulate our syntactic descriptions as follows:

THEOREM 1.5 The pseudovariety $\mathrm{T}_{p}^{q}$ of all finite semigroups triangularizable over the field of characteristic $p$ and order $q$ is defined by the pseudoidentities

$$
\begin{gathered}
\left((\text { exe })^{q-1}(\text { eye })^{q-1}\right)^{p^{\omega}}=e, \\
\left((\text { exe })^{\omega-1}(\text { eye })^{\omega-1} \text { exeye }\right)^{p^{\omega}}=e, \\
\left(e(y x)^{\omega} e\right)^{\omega}=e, \\
\left((e x)^{p^{\omega}-1}(y e)^{p^{\omega}-1}\right)^{p^{\omega}+1}=(e x)^{p^{\omega}-1}(y e)^{p^{\omega}-1} .
\end{gathered}
$$

COROLlary 1.6 The pseudovariety $\mathrm{T}_{p}$ of all finite semigroups triangularizable over a finite field of characteristic $p$ is defined by the pseudoidentities (1.9)(1.11) and

$$
\left((\text { exe })^{p^{\omega}-1}(e y e)^{p^{\omega}-1}\right)^{p^{\omega}}=e .
$$


All the pseudoidentities (1.8)-(1.12) depend on two variables. Clearly, if one evaluates the left hand side and the right hand side of such a pseudoidentity in a finite semigroup $S$ assigning the variables $x$ and $y$ some elements $s, t \in S$, then the evaluation takes place in the subsemigroup of $S$ generated by $s$ and $t$. Therefore we have the following corollary:

COROllary 1.7 A finite semigroup $S$ is triangularizable over a given finite field [over some finite field of a given characteristic] whenever each of its 2generator subsemigroups is so.

As was pointed out to the authors by one of the referee's, this corollary can be also obtained by combining the fact (following from Theorem 1.2 and Corollary 1.3) that triangularizability of a semigroup over a finite field only depends on its isomorphism class with a classical result of Guralnick [10].

The proofs in this paper are designed to be self-contained; in particular, we do not assume the reader's acquaintance with the representation theory of finite semigroups or with the theory of linear groups using arguments from elementary linear algebra instead. In fact, a reader having such a background may observe that some of the proofs can be shortened by representation-theoretical arguments.

\section{Preliminaries on matrix semigroups}

We fix a (not necessarily finite) field $K$ and denote by $M_{n}(K)$ the semigroup of all $n \times n$ matrices over $K$. Let $T_{n}(K)$ denote the semigroup of all upper triangular matrices from $M_{n}(K)$, and let $U T_{n}(K)$ stand for its subsemigroup consisting of unitriangular matrices. The next proposition collects some properties of the regular $\mathscr{D}$-classes and the maximal subgroups of $T_{n}(K)$; it plays a crucial role in the proof of the 'only if' part of Proposition 1.1 and Theorem 1.2. For proofs of all these properties the reader is referred to [16, Section 3.2, Example 2]. Of course, in this paper we are especially interested in the case of a finite field; for this special case, direct and elementary proofs of most of the claims of the proposition may be found in [30].

Proposition 2.1 In the semigroup $T_{n}(K)$ every regular $\mathscr{D}$-class consists of all matrices of rank $j, 0 \leq j \leq n$, whose main diagonals have exactly $j$ nonzero entries and the same pattern of zero entries. Each such regular $\mathscr{D}$-class is a subsemigroup and hence the union of its maximal subgroups. Each maximal subgroup $H$ of a regular $\mathscr{D}$-class whose matrices have rank $j$ is isomorphic to the group $T_{j}^{*}(K)$ of all invertible matrices of $T_{j}(K)$, and the group $H \cap U T_{n}(K)$ is isomorphic to the group $U T_{j}^{*}(K)$ of all invertible matrices of $U T_{j}(K)$.

Our proofs of the "if" part of Theorem 1.2 and of Theorem 1.5 depend heavily on Okniński's triangularizability criterion [16, Section 4.4] or, more precisely, on its restriction to the case of finite matrix semigroups (over an arbitrary field). Its formulation requires a few definitions.

We say that two subsemigroups $S$ and $T$ of $M_{n}(K)$ are conjugate if there exists an invertible matrix $g \in M_{n}(K)$ such that $g^{-1} S g=T$. A matrix is called unipotent if all its eigenvalues belong to the set $\{0,1\}$. 
Proposition 2.2 A finite subsemigroup $S \subseteq M_{n}(K)$ is conjugate to a subsemigroup in $T_{n}(K)$ if and only if every maximal subgroup of $S$ is conjugate to a subgroup in $T_{n}(K)$, every regular $\mathscr{D}$-class $D$ of $S$ is a subsemigroup of $S$, and the subsemigroup of $D$ generated by its idempotents consists of unipotent matrices.

Okniński's triangularizability criterion [16, Theorem 4.31] for an arbitrary matrix semigroup is formulated in a similar way; the only difference is that instead of regular $\mathscr{D}$-classes Okniński uses a more general notion of a uniform component of a matrix semigroup. However it easily follows from [16, Section 3.2, Remark v)] that the uniform components of a finite semigroup $S$ are just the regular $\mathscr{D}$-classes of $S$.

We also make use of the following classic result by Kolchin [13, Section 1]:

Proposition 2.3 Every subgroup in $M_{n}(K)$ consisting of unipotent matrices is conjugate to a subgroup in $T_{n}(K)$.

Combining Propositions 2.2 and 2.3 one immediately gets the following corollary which is a restriction of [16, Corollary 4.33] to the finite case:

Corollary 2.4 A finite subsemigroup $S \subseteq M_{n}(K)$ consisting of unipotent matrices is conjugate to a subsemigroup of $T_{n}(K)$ if and only if the regular $\mathscr{D}$-classes of $S$ are subsemigroups.

In order to apply Proposition 2.2 or Corollary 2.4 to an abstract semigroup $S$ we have to faithfully represent $S$ by matrices over the given finite field. The reader may think that this makes no real problem because it is well known that every finite semigroup admits a faithful linear representation over any field $K$. (It suffices to span a $K$-vector space by the set $S^{1}$ and then extend the regular representation of $S$ to this space.) A difficulty still remains because in Proposition 2.2 and Corollary 2.4 the notion of unipotency refers to a property of a given linear representation of $S$ rather than semigroup properties of $S$. However, the results of the present paper show that triangularizability of a matrix semigroup over a finite field depends only on abstract properties of the semigroup. The key observation here is the following easy lemma that, for a matrix $a$ over a finite field, expresses the property of being unipotent as a property of the period of the cyclic subsemigroup $a$ generates.

Lemma 2.5 A matrix a over a finite field of characteristic $p$ is unipotent if and only if $a^{k}=a^{k+p^{\ell}}$ for some positive integers $k$ and $\ell$.

Proof. For the 'only if' part, consider a triangular conjugate $t$ of the matrix $a$ (it exists because the eigenvalues of $a$ belong to the ground field). Then $t$ is a unitriangular matrix, and a straightforward calculation shows that any unitriangular matrix over a finite field of characteristic $p$ satisfies $t^{k}=t^{k+p^{\ell}}$ for some $k$ and $\ell$.

For the 'if' part, let $K$ be the splitting field of the characteristic polynomial of $a$ and $\lambda \in L$ an eigenvalue of $a$. Then $\lambda$ satisfies the equation $\lambda^{k}=\lambda^{k+p^{\ell}}$. Thus,

$$
0=\lambda^{k+p^{\ell}}-\lambda^{k}=\lambda^{k}\left(\lambda^{p^{\ell}}-1\right)=\lambda^{k}(\lambda-1)^{p^{\ell}}
$$


since $p$ is the characteristic of the field $K$. We conclude that either $\lambda=0$ or $\lambda=1$, that is, $a$ is a unipotent matrix.

\section{Preliminaries on groups}

We need a few facts about finite groups that all are slight variations of well known results (see, e.g., [9, Chapter 3]). It appears however that no source in the vast group-theoretic literature contains these facts in a form suitable for use in the present paper. Therefore, for the sake of being reasonably self-contained and for the reader's convenience, we provide their detailed formulations supplied with elementary proofs.

We start with justifying the pseudoidentity bases for the pseudovarieties $\mathrm{G}_{p} A b^{q-1}$ and $\mathrm{G}_{p} \mathrm{Ab}$ presented in Section 1 .

Lemma 3.1 The pseudovariety $\mathrm{G}_{p} \mathrm{Ab}^{q-1}$ is defined within the class of all finite groups by the pseudoidentities (1.4) and (1.5). The pseudovariety $\mathrm{G}_{p} \mathrm{Ab}$ is defined within the class of all finite groups by the pseudoidentities (1.6) and (1.5).

Proof. First we prove that every group $G \in \mathrm{G}_{p} \mathrm{Ab}^{q-1}$ satisfies the pseudoidentities (1.4) and (1.5). By the definition of $\mathrm{G}_{p} \mathrm{Ab}^{q-1}$, such $G$ is an extension of a $p$-subgroup $H$ by an Abelian group of exponent $q-1$. Therefore for any $x, y \in G$, we have $x^{q-1} y^{q-1} \in H$ and $x^{-1} y^{-1} x y \in H$. If $|G|=\ell$, then $g^{\ell !}=1$ for all $g \in G$ whence $g^{-1}=g^{\ell !-1}$. Further, $h^{p^{\ell !}}=1$ for all $h \in H$. Combining all these observations, we conclude that $G$ satisfies

$$
\left(x^{q-1} y^{q-1}\right)^{p^{\ell !}}=1 \text { and }\left(x^{\ell !-1} y^{\ell !-1} x y\right)^{p^{\ell !}}=1 .
$$

By our interpretation of $\omega$-words, this means that $G$ satisfies (1.4) and (1.5).

Now take $G \in \mathrm{G}_{p} \mathrm{Ab}$. Clearly, the above proof applies to show that $G$ satisfies (1.5). By the definition of $\mathrm{G}_{p} \mathrm{Ab}$, the commutator subgroup $G^{\prime}$ of $G$ is a $p$-group. Every $p$-subgroup of $G$ is contained in a Sylow $p$-subgroup but since subgroups containing $G^{\prime}$ are all normal in $G$, we conclude that $G$ has a unique Sylow $p$-subgroup $H$ which is normal in $G$. The quotient $G / H$ is an Abelian group whose order $r$ is relatively prime with $p$. Then by Euler's theorem, $r$ divides $p^{m}-1$ for a suitable $m<r$. If $|G|=\ell$, then $m$ divides $\ell$ ! whence $p^{m}-1$ divides $p^{\ell !}-1$ and we have $x^{p^{\ell !}-1} y^{p^{\ell !}-1} \in H$ for all $x, y \in G$. As in the previous paragraph, we conclude that $G$ satisfies

$$
\left(x^{p^{\ell !}-1} y^{p^{\ell !}-1}\right)^{p^{\ell !}}=1
$$

and hence $G$ satisfies the pseudoidentity (1.6).

Now suppose that $G$ is a group of order $\ell$ satisfying (1.4) and (1.5). According to our convention, the $\omega$-words $\left(x^{q-1} y^{q-1}\right)^{p^{\omega}}$ and $\left(x^{\omega-1} y^{\omega-1} x y\right)^{p^{\omega}}$ interpret in $G$ as $\left(x^{q-1} y^{q-1}\right)^{p^{p !}}$ and respectively $\left(x^{\ell !-1} y^{\ell !-1} x y\right)^{p^{\ell !}}$. In particular, $G$ satisfies the identity

$$
\left(x^{q-1} y^{q-1}\right)^{p^{\ell !}}=1 \text {. }
$$


Recall that $q$ is a power of $p$, and therefore, the numbers $q-1$ and $p^{\ell !}$ are relatively prime. It is known (cf. [15, Example 34.25]) and easy to verify that in every group satisfying the identity $\left(x^{s} y^{s}\right)^{r}=1$ for some relatively prime numbers $s$ and $r$ the elements of order dividing $r$ form a normal subgroup. We see that $G$ has a normal $p$-subgroup $H$ such that the quotient group $G / H$ satisfies $x^{q-1}=1$ and $x^{\ell !-1} y^{\ell !-1} x y=1$. Since the exponent of any group of order $\leq \ell$ divides $\ell$ !, we have $x^{\ell !}=1$ in $G / H$ whence $x^{\ell !-1}=x^{-1}$ and the expression $x^{\ell !-1} y^{\ell !-1} x y$ is nothing but the usual group commutator of the elements $x$ and $y$. Thus, we have $G / H \in \mathrm{Ab}^{q-1}$ and $G \in \mathrm{G}_{p} \mathrm{Ab}^{q-1}$, as required.

The same proof with $p^{\ell !}$ in the role of $q$ shows that every finite group satisfying (1.6) and (1.5) belongs to the pseudovariety $\mathrm{G}_{p} \mathrm{Ab}$.

We notice that the proof of Lemma 3.1 also justifies the following formula:

$$
\mathrm{G}_{p} \mathrm{Ab}=\bigcup_{m} \mathrm{G}_{p} \mathrm{Ab} \mathrm{b}^{p^{m}-1} .
$$

Now we aim to verify that every subgroup of $M_{n}(\mathbb{F})$ that belongs to the pseudovariety $\mathrm{G}_{p} \mathrm{Ab}^{q-1}$ is conjugate to a subgroup in $T_{n}(\mathbb{F})$. We start with a reduction which, though it is not essential, allows us to simplify the notation. Let $G$ be a subgroup in $M_{n}(\mathbb{F})$ and let $e$ be the identity element of $G$. It is well known (and easy to verify) that the semigroup $e M_{n}(\mathbb{F}) e$ is isomorphic to $M_{k}(\mathbb{F})$ where $k$ is the rank of the matrix $e$. The group $G \subseteq e M_{n}(\mathbb{F}) e$ is then isomorphic to a subgroup of the group $G L_{k}(\mathbb{F})$ of all invertible matrices in $M_{k}(\mathbb{F})$. In other words, we may (and will) assume that groups under consideration consist of invertible matrices.

Given a subgroup $G \subseteq G L_{n}(\mathbb{F})$, we denote by $C(G)$ the following subspace of the vector space $\mathbb{F}^{n}$ :

$$
C(G)=\left\{v \in \mathbb{F}^{n} \mid v g_{1} g_{2}=v g_{2} g_{1} \text { for all } g_{1}, g_{2} \in G\right\} .
$$

Lemma 3.2 The subspace $C(G)$ is $G$-invariant.

Proof. Take an arbitrary vector $v \in C(G)$ and an arbitrary matrix $h \in G$. Then for all $g_{1}, g_{2} \in G$ we have

$$
(v h) g_{1} g_{2}=v h\left(g_{1} g_{2}\right)=v g_{1} g_{2} h=v g_{2} g_{1} h=v h\left(g_{2} g_{1}\right)=(v h) g_{2} g_{1}
$$

whence $v h \in C(G)$.

Lemma 3.3 If a subgroup $G \subseteq G L_{n}(\mathbb{F})$ belongs to the pseudovariety $\mathrm{G}_{p} \mathrm{Ab}$, then $C(G) \neq 0$.

Proof. By Lemma 2.5 the commutator subgroup $G^{\prime}$ of $G$ consists of unipotent matrices. Since $G \subseteq G L_{n}(\mathbb{F})$, the only eigenvalue for all matrices in $G^{\prime}$ is 1 . By Proposition 2.3 (Kolchin's theorem) all matrices in $G^{\prime}$ possess a common eigenvector $v \neq 0$, so $v h=v$ for every $h \in G^{\prime}$. Now for all $g_{1}, g_{2} \in G$ we have $g_{1} g_{2} g_{1}^{-1} g_{2}^{-1} \in G^{\prime}$ whence

$$
v g_{1} g_{2}=v\left(g_{1} g_{2} g_{1}^{-1} g_{2}^{-1}\right) g_{2} g_{1}=v g_{2} g_{1} .
$$

We see that $v \in C(G)$, and hence, $C(G) \neq 0$. 
Proposition 3.4 If a subgroup $G \subseteq G L_{n}(\mathbb{F})$ belongs to the pseudovariety $\mathrm{G}_{p} \mathrm{Ab}$, then there exists a chain of $G$-invariant subspaces

$$
0=C_{0} \subset C_{1} \subset \cdots \subset C_{k-1} \subset C_{k}=\mathbb{F}^{n}
$$

such that for each $i=1, \ldots, k$, the group $G_{i}$ of invertible linear transformations induced by $G$ on the quotient space $C_{i} / C_{i-1}$ is Abelian.

Proof. We build the chain (3.2) inductively. Suppose the subspace $C_{i-1}$ is already defined and consider the quotient space $\mathbb{F}^{n} / C_{i-1}$. Since $C_{i-1}$ is a $G$-invariant subspace, the group $G$ induces a group $H_{i}$ of invertible linear transformations of the space $\mathbb{F}^{n} / C_{i-1}$. Clearly, $H_{i}$ is a homomorphic image of $G$, and therefore, $H_{i}$ belongs to the pseudovariety $\mathrm{G}_{p} \mathrm{Ab}$. By Lemma 3.3 the subspace $C\left(H_{i}\right)$ of $\mathbb{F}^{n} / C_{i-1}$ is not equal to 0 and by Lemma 3.2 it is $H_{i^{-}}$ invariant. Now let $C_{i}$ be the pullback of $C\left(H_{i}\right)$ in $\mathbb{F}^{n}$. Then $C_{i-1} \subset C_{i}$ and $C_{i}$ is a $G$-invariant subspace. By the definition of the subspace $C\left(H_{i}\right)$, the group $G_{i}$ formed by the restrictions of the transformations of $H_{i}$ to this subspace is Abelian. The second isomorphism theorem implies that $G_{i}$ is isomorphic to the group of invertible linear transformations induced by $G$ on the quotient space $C_{i} / C_{i-1}$.

A matrix form of Proposition 3.4 is provided by the following

COROLlary 3.5 If a subgroup of $G L_{n}(\mathbb{F})$ lies in the pseudovariety $G_{p} A b$, then it is conjugate to a group $G$ of the form

$$
\left(\begin{array}{ccccc}
G_{k} & * & \ldots & * & * \\
0 & G_{k-1} & \ldots & * & * \\
\vdots & \vdots & \ddots & \vdots & \vdots \\
0 & 0 & \ldots & G_{2} & * \\
0 & 0 & \ldots & 0 & G_{1}
\end{array}\right)
$$

Here each $G_{i}(i=1, \ldots, k)$ is an Abelian group of $n_{i} \times n_{i}$ matrices where $n_{1}+\cdots+n_{k}=n$.

Proof. It suffices to choose a basis $v_{1}, \ldots, v_{n}$ for the space $\mathbb{F}^{n}$ according to the chain (3.2), i.e. choose it so that $v_{1}, \ldots, v_{n_{1}}$ is a basis of $C_{1}, v_{n_{1}+1}+$ $C_{1}, \ldots, v_{n_{1}+n_{2}}+C_{1}$ is a basis of $C_{2} / C_{1}$, etc.

Recall that $q$ stands for the order of the field $\mathbb{F}$.

Proposition 3.6 If a subgroup $G \subset M_{n}(\mathbb{F})$ belongs to the pseudovariety $\mathrm{G}_{p} \mathrm{Ab}^{q-1}$, then $G$ is conjugate to a subgroup of $T_{n}(\mathbb{F})$.

Proof. If we take $G$ in the block-triangular form of Corollary 3.5, it remains to verify that each diagonal group $G_{i}$ is conjugate to a subgroup of $T_{n_{i}}(\mathbb{F})$. In other words, we may assume that $G$ is Abelian.

Now take an arbitrary matrix $g \in G$, let $K \supseteq \mathbb{F}$ be the splitting field of the characteristic polynomial of $g$ and let $\lambda \in K$ be an arbitrary eigenvalue of $g$. 
Since $G$ satisfies the pseudoidentity (1.4), $g^{(q-1) p^{\ell !}}=1$ where $\ell=|G|$. Hence $\lambda$ satisfies the equation $\lambda^{(q-1) p^{\ell !}}=1$. On the other hand, $\lambda^{q^{m}-1}=1$ where $q^{m}$ is the order of the field $K$. Since $q$ is a power of $p$, the greatest common divisor of $(q-1) p^{\ell !}$ and $q^{m}-1=(q-1)\left(q^{m-1}+\cdots+q+1\right)$ is equal to $q-1$. Thus, $\lambda^{q-1}=1$, and this means that $\lambda$ (as a solution to the equation $x^{q}=x$ over $K$ ) belongs to the field $\mathbb{F}$.

It remains to recall the following elementary exercise in linear algebra: every finite family of commuting matrices whose eigenvalues lie in the ground field is simultaneously triangularizable over that field.

From Proposition 3.6 and the formula (3.1) we immediately obtain

COROLlary 3.7 Every group $G$ in the pseudovariety $\mathrm{G}_{p} \mathrm{Ab}$ is triangularizable over a suitable finite field of characteristic $p$.

\section{Proofs of the main results}

We start by proving Theorem 1.2. Recall that it claims that the class $\mathbf{T}_{p}^{q}$ of all finite semigroups triangularizable over the field $\mathbb{F}$ of characteristic $p$ and order $q$ is the pseudovariety $\overline{\mathrm{G}_{p} \mathrm{Ab} \mathrm{b}^{q-1}} \cap \mathrm{DS} \cap \mathcal{E} \overline{\mathrm{G}_{p}}$.

Necessity. It suffices to verify that for each $n$ the semigroup $T_{n}(\mathbb{F})$ belongs to $\overline{\mathrm{G}_{p} \mathrm{Ab}^{q-1}} \cap \mathrm{DS} \cap \mathcal{E} \overline{\mathrm{G}_{p}}$. The fact that $T_{n}(\mathbb{F}) \in \mathrm{DS}$ is a part of Proposition 2.1.

By the same proposition the maximal subgroups of $T_{n}(\mathbb{F})$ are of the form $T_{j}^{*}(\mathbb{F})$ for some $j \leq n$. The mapping that sends every matrix of the group $T_{j}^{*}(\mathbb{F})$ to the diagonal matrix with the same diagonal elements is a homomorphism whose kernel is $U T_{j}^{*}(\mathbb{F})$ and whose image (that is, the group of all invertible diagonal $j \times j$ matrices) is isomorphic to the direct product of $j$ copies of the multiplicative group of the field $\mathbb{F}$. The group $U T_{j}^{*}(\mathbb{F})$ is easily seen to be a $p$-group (indeed, the subgroup contains $q^{\frac{j(j-1)}{2}}$ elements, and this number is a power of $p$ ). Thus, $T_{j}^{*}(\mathbb{F})$ is an extension of a $p$-group by an Abelian group of exponent $q-1$. We see that every subgroup of the semigroup $T_{n}(\mathbb{F})$ belongs to the pseudovariety $\mathrm{G}_{p} A b^{q-1}$ whence the semigroup itself lies in $\overline{\mathrm{G}_{p} A b^{q-1}}$.

Clearly, the idempotents of $T_{n}(\mathbb{F})$ belong to the semigroup $U T_{n}(\mathbb{F})$ which lies in the pseudovariety $\overline{\mathrm{G}_{p}}$ since its maximal subgroups are $p$-groups, see Proposition 2.1. Therefore $T_{n}(\mathbb{F}) \in \mathcal{E} \overline{\mathrm{G}_{p}}$.

Sufficiency. Take a finite semigroup $S \in \overline{\mathrm{G}_{p} \mathrm{Ab}^{q-1}} \cap \mathrm{DS} \cap \mathcal{E} \overline{\mathrm{G}_{p}}$. As discussed in Section 2, we may assume that $S \subseteq M_{n}(\mathbb{F})$ for some $n$. Now we aim to show that $S$ satisfies the conditions of Proposition 2.2.

The fact that every maximal subgroup of $S$ is conjugate to a subgroup in $T_{n}(\mathbb{F})$ follows from Proposition 3.6. Since $S \in \mathrm{DS}$, each regular $\mathscr{D}$-class $D$ is a subsemigroup of $S$ and it remains to check that the subsemigroup of $D$ generated by its idempotents consists of unipotent matrices. But, since $S$ lies in $\mathcal{E} \overline{\mathrm{G}}_{p}$, the idempotents of $S$ (and, in particular, the idempotents of $D$ ) generate a subsemigroup that belongs to $\overline{\mathrm{G}_{p}}$. By Lemma 2.5 this subsemigroup consists of unipotent matrices. 
Now it is easy to prove Proposition 1.1. We recall its assertion: the class $\mathrm{UT}_{p}$ of all finite semigroups unitriangularizable over a given finite field of characteristic $p$ coincides with $\overline{\mathrm{G}_{p}} \cap \mathrm{DS}$.

Necessity follows from Proposition 2.1.

Sufficiency follows from Corollary 2.4 and from the above proof of the 'if' part of Theorem 1.2. In order to see that all elements of the semigroup are unipotent, one should take into account the pseudoidentity (1.3) defining the pseudovariety $\overline{\mathrm{G}_{p}}$ and use Lemma 2.5 .

In order to obtain Corollary 1.3, one repeats the proof of Theorem 1.2 using Corollary 3.7 instead of Proposition 3.6.

Next we prove Theorem 1.5 that claims that the pseudovariety $\mathrm{T}_{p}^{q}$ can be defined by the pseudoidentities (1.8)-(1.11). First we show that $\mathrm{T}_{p}^{q}$ satisfies these 4 pseudoidentities. To this aim it suffices to verify that they hold in the semigroup $T_{n}(\mathbb{F})$ for each $n$.

Clearly, $T_{n}(\mathbb{F})$ satisfies the pseudoidentity (1.10) because this pseudoidentity defines the pseudovariety DS and $T_{n}(\mathbb{F})$ belongs to DS by Proposition 2.1.

Now we take two arbitrary matrices $x, y \in T_{n}(\mathbb{F})$ and let $\ell=\left|T_{n}(\mathbb{F})\right|$. The $\omega$-word $(x y)^{\omega}$ interprets in $T_{n}(\mathbb{F})$ as $(x y)^{\ell !}$, and this is an idempotent in $T_{n}(\mathbb{F})$. Slightly abusing notation, we denote the idempotent by $e$. Then the matrices $e x$ and ye belong to the same regular $\mathscr{D}$-class $D$ of $S$ : indeed, one readily sees that the element $e x$ is $\mathscr{R}$-related to the idempotent $e$ while the element ye is $\mathscr{L}$-related to the same idempotent. Since $D$ is a subsemigroup, we have exe, eye $\in D$. The idempotent $e$ is the identity for both exe and eye whence the two elements belong to the $\mathscr{H}$-class of $e$ which is a group. Subgroups of $T_{n}(\mathbb{F})$ belong to the pseudovariety $\mathrm{G}_{p} \mathrm{Ab}^{q-1}$ by Theorem 1.2. By Lemma 3.1 this pseudovariety satisfies the pseudoidentities (1.4) and (1.5). Thus we have that the equalities

$$
\begin{gathered}
\left((\text { exe })^{q-1}(\text { eye })^{q-1}\right)^{p^{\ell !}}=e, \\
\left((\text { exe })^{\ell !-1}(\text { eye })^{\ell !-1} \text { exeye }\right)^{p^{\ell !}}=e
\end{gathered}
$$

hold in $T_{n}(\mathbb{F})$ for an arbitrary choice of the elements $x$ and $y$.

Further, by Proposition 2.1 the matrices $e x$ and ye are of the same rank $j$, say, and their main diagonals have exactly $j$ non-zero entries and the same pattern of zero entries. Since $q$ is a power of $p$ and $q \leq \ell$, the number $q-1$ divides $p^{\ell !}-1$ and the non-zero diagonal entries of the matrices $(e x)^{p^{\ell !}-1}$ and $(y e)^{p^{\ell !}-1}$ are equal to 1 . The product $z$ of the two matrices belongs to $D$ (since $D$ is a subsemigroup), and therefore, $z$ is a group element (since $D$ is a union of its maximal subgroups). At the same time $z$ is a unitriangular matrix. We already have observed that subgroups of $U T_{n}(\mathbb{F})$ are $p$-groups whence $z$ belongs to a $p$-group of order $\leq \ell$ and thus satisfies $z^{p^{\ell !}+1}=z$.

By our interpretation of $\omega$-words, this means that $T_{n}(\mathbb{F})$ satisfies (1.8), (1.9) and (1.11). 
For the converse, we have to prove that if a finite semigroup $S$ satisfies the pseudoidentities (1.8)-(1.11), then $S$ belongs to the pseudovariety $\mathrm{T}_{p}^{q}$, that is, $S$ is triangularizable over the field $\mathbb{F}$. As in the proof of the 'if' part of Theorem 1.2, we may assume that $S \subseteq M_{n}(\mathbb{F})$ for some $n$. We then have to show that $S$ satisfies the conditions of Proposition 2.2.

First, let $G$ be a subgroup of $S$ and $x, y \in G$. Then the $\omega$-word $e=(x y)^{\omega}$ interprets in $S$ as the identity element of $G$ whence exe and eye interpret as respectively $x$ and $y$. Therefore the fact that $S$ satisfies the pseudoidentities (1.8) and (1.9) implies that $G$ satisfies the pseudoidentities (1.4) and respectively (1.5). By Lemma 3.1 the latter pseudoidentities define the pseudovariety $\mathrm{G}_{p} \mathrm{Ab}^{q-1}$ and so $G$ lies in this pseudovariety. By Proposition $3.6 G$ is conjugate to a subgroup in $T_{n}(\mathbb{F})$.

The fact that every regular $\mathscr{D}$-class of $S$ is a subsemigroup is ensured since $S$ satisfies the pseudoidentity (1.10).

To complete the proof we need to show that, for every regular $\mathscr{D}$-class $D$ of $S$, the subsemigroup of $D$ generated by its idempotents consists of unipotent matrices.

Let $\ell=|S|$ and let $g \in D$ be a unipotent matrix. Since $D$ is a union of groups, $g$ belongs to a subgroup whence the cyclic subsemigroup generated by $g$ is in fact a subgroup. By Lemma 2.5 the cyclic subgroup is in fact a $p$-subgroup whose identity element can be then written as $g^{p^{\ell !}}$. Hence the inverse $\bar{g}$ of $g$ in the subgroup is equal to $g^{p^{\ell !}-1}$. Therefore $g=\bar{g}^{p^{\ell !}-1}$. Now let $h \in D$ be another unipotent matrix and $\bar{h}$ its inverse in the corresponding subgroup of $D$. It is well-known that the pseudovariety of all finite simple semigroups is defined by the pseudoidentity $(x y)^{\omega} x=x$. Hence, as a finite simple semigroup of order at most $\ell, D$ satisfies the identity $(x y)^{\ell !} x=x$. Thus, $(\bar{g} \bar{h})^{\ell !} \bar{g}=\bar{g}$ and $\bar{h}(\bar{g} \bar{h})^{\ell !}=\bar{h}$ whence

$$
\left((\bar{g} \bar{h})^{\ell !} \bar{g}\right)^{p^{\ell !}-1}\left(\bar{h}(\bar{g} \bar{h})^{\ell !}\right)^{p^{\ell !}-1}=g h .
$$

In view of the pseudoidentity $(1.7)$ we get $g h=(g h)^{p^{\ell !}+1}$ and by Lemma 2.5 $g h$ is a unipotent matrix. Thus, we have proved that the product of any two unipotent matrices from a regular $\mathscr{D}$-class is again a unipotent matrix from the same $\mathscr{D}$-class.

Since it is clear that every idempotent matrix is unipotent, it follows from the previous paragraph by a straightforward induction that the idempotentgenerated subsemigroup of $D$ consists of unipotent matrices, and this completes the proof of Theorem 1.5.

The proof of Corollary 1.6 is completely analogous to the above proof of Theorem 1.5.

\section{An example}

In order to illustrate some subtleties, we analyze an easy but rather peculiar example in which three 24-element simple semigroups sharing the same structure group behave in a completely different manner with respect to triangularizability over a finite field. 
Let $\mathbb{Z}_{6}=\left\langle c \mid c^{7}=c\right\rangle$ stand for the cyclic group of order 6 , and let $e=c^{6}$ be the identity of this group. Now consider the following three matrices over $\mathbb{Z}_{6}$ :

$$
P_{1}=\left(\begin{array}{cc}
e & e \\
e & c
\end{array}\right), \quad P_{2}=\left(\begin{array}{cc}
e & e \\
e & c^{2}
\end{array}\right), \quad P_{3}=\left(\begin{array}{cc}
e & e \\
e & c^{3}
\end{array}\right)
$$

EXAmple 5.1 Let $M\left(\mathbb{Z}_{6}, P_{i}\right)(i=1,2,3)$ be the Rees matrix semigroup over $\mathbb{Z}_{6}$ with the sandwich matrix $P_{i}$.

(i) The semigroup $M\left(\mathbb{Z}_{6}, P_{1}\right)$ is triangularizable over no finite field.

(ii) The semigroup $M\left(\mathbb{Z}_{6}, P_{2}\right)$ is triangularizable over a finite field if and only if the field has characteristic 3.

(iii) The semigroup $M\left(\mathbb{Z}_{6}, P_{3}\right)$ is triangularizable over a finite field if and only if the field consists of $4^{m}$ elements.

Proof. It is easy to calculate (and also follows from some general properties of Rees matrix semigroups) that the maximal subgroups of the subsemigroup generated by the idempotents of $M\left(\mathbb{Z}_{6}, P_{i}\right)$ are isomorphic to the subgroup $H_{i}$ of $\mathbb{Z}_{6}$ generated by the element $c^{i}, i=1,2,3$. For $i=1$, the group $H_{1}=\mathbb{Z}_{6}$ is not a $p$-group for any prime $p$. Therefore there is no prime $p$ such that $M\left(\mathbb{Z}_{6}, P_{1}\right)$ can belong to $\mathcal{E} \overline{\mathrm{G}_{p}}$ and by Theorem 1.2 the semigroup cannot be faithfully represented by triangular matrices over any finite field.

For $i=2$, the group $H_{2}$ has order 3 whence $M\left(\mathbb{Z}_{6}, P_{2}\right)$ belongs to $\mathcal{E} \overline{\mathrm{G}_{3}}$. Since the semigroup obviously lies in DS and its maximal subgroup $\mathbb{Z}_{6}$ can be treated as an extension of a 3-group by an Abelian group of exponent $2=3-1$, Theorem 1.2 applies with $p=q=3$. We see that $M\left(\mathbb{Z}_{6}, P_{2}\right)$ is triangularizable over the 3 -element field and hence over any finite field of characteristic 3 (but over no finite field of other characteristic).

If $i=3$, then the group $H_{3}$ has order 2. Thus, $M\left(\mathbb{Z}_{6}, P_{3}\right)$ belongs to $\mathcal{E} \overline{\mathrm{G}_{2}} \cap \mathrm{DS}$. This time we should treat $\mathbb{Z}_{6}$ as an extension of a 2 -group by an Abelian group of exponent $3=4-1$. By Theorem $1.2($ with $p=2, q=4$ ) the semigroup $M\left(\mathbb{Z}_{6}, P_{3}\right)$ is triangularizable over the 4-element field and hence over any field with $4^{m}$ elements. On the other hand, if a finite field $\mathbb{F}$ does not contain the 4-element field, then either its characteristic is not 2 or its multiplicative group has no elements of order 3 , and therefore, $M\left(\mathbb{Z}_{6}, P_{3}\right)$ is not triangularizable over $\mathbb{F}$.

The following 'egg-box picture' demonstrates a concrete faithful representation of the semigroup $M\left(\mathbb{Z}_{6}, P_{2}\right)$ by $3 \times 3$ triangular matrices of the 3 -element field $\mathbb{Z} / 3 \mathbb{Z}$ (this representation is in fact of the minimum possible degree). We have filled only in the upper triangle of the matrices; all blank positions are assumed to be filled with zeros. 


$$
\begin{aligned}
& \left(\begin{array}{lll}
1 & 0 & 0 \\
& 0 & 0 \\
& & 1
\end{array}\right) \\
& \left(\begin{array}{lll}
2 & 0 & 0 \\
& 0 & 0 \\
& & 2
\end{array}\right)
\end{aligned}
$$

A similar minimum representation by $3 \times 3$ triangular matrices of the 4 element field can be constructed for the semigroup $M\left(\mathbb{Z}_{6}, P_{3}\right)$.

Acknowledgments. Several useful comments of the anonymous referees are gratefully acknowledged.

\section{References}

[1] J. Almeida, Implicit operations on finite $\mathscr{J}$-trivial semigroups and a conjecture of I. Simon, J. Pure Appl. Algebra 69 (1990) 205-218.

[2] J. Almeida, Finite Semigroups and Universal Algebra, World Scientific, 1995.

[3] J. Almeida and A. Azevedo, Globals of pseudovarieties of commutative semigroups: the finite basis problem, decidability, and gaps, Proc. Edinburgh Math. Soc. 44 (2001) 27-47.

[4] J. Almeida and M. V. Volkov, Profinite identities for finite semigroups whose subgroups belong to a given pseudovariety, J. Algebra and its Applications 2 (2003) 137-163.

[5] A. H. Clifford and G. B. Preston, The Algebraic Theory of Semigroups, Amer. Math. Soc., Vol. I, 1961; Vol. II, 1967.

[6] R. S. Cohen and J. A. Brzozowski, Dot-depth of star-free events, J. Comp. System Sci. 5 (1971) 1-15.

[7] S. Eilenberg, Automata, Languages and Machines, Academic Press, Vol. A, 1974; Vol. B, 1976.

[8] S. Eilenberg and M. P. Schützenberger, On pseudovarieties, Adv. Math. 19 (1976) 413-418.

[9] D. Gorenstein, Finite Groups, 2nd edition, Chelsea Publishing Company, 1980.

[10] R. M. Guralnick, Triangularization of sets of matrices, Linear and Multilinear Algebra 9 (1980) 133-140.

[11] K. Henckell and J.-E. Pin, Ordered monoids and $\mathscr{J}$-trivial monoids, in J.-C. Birget, S. Margolis, J. Meakin, M. Sapir (eds.), Algorithmic problems in groups and semigroups, Birkhäuser, 2000, 121-137. 
[12] P. Higgins, A proof of Simon's theorem on piecewise testable languages, Theor. Comp. Sci. 178 (1997) 257-264.

[13] E. R. Kolchin, On certain concepts in the theory of algebraic matrix groups, Ann. Math. 49 (1948) 774-789.

[14] G. Lallement, Semigroups and Combinatorial Applications, John Wiley \& Sons, 1979.

[15] H. Neumann, Varieties of groups, Springer-Verlag, 1967.

[16] J. Okniński, Semigroup of Matrices, World Scientific, 1998.

[17] J-E. Pin, Variétés de langages formels, Masson, 1984 [French; Engl. translation Varieties of formal languages, North Oxford Academic, 1986 and Plenum, 1986].

[18] J-E. Pin and H. Straubing, Monoids of upper triangular matrices, in G. Pollák, Št. Schwarz and O. Steinfeld (eds.), Semigroups. Structure and Universal Algebraic Problems [Colloquia Mathematica Societatis János Bolyai 39], North-Holland, 1985, 259-272.

[19] H. Radjavi and P. Rosenthal, Simultaneous Triangularization, Springer-Verlag, 2000.

[20] J. Reiterman, The Birkhoff theorem for finite algebras, Algebra Universalis 14 (1982) 1-10.

[21] I. Simon, Hierarchies of Events of Dot-Depth One, Ph. D. Thesis, University of Waterloo, 1972.

[22] I. Simon, Piecewise testable events, Proc. 2nd GI Conf. [Lect. Notes Comp. Sci. 33], Springer-Verlag, 1975, 214-222.

[23] J. Stern, Characterization of some classes of regular events, Theor. Comp. Sci. 35 (1985) 17-42.

[24] H. Straubing, On finite $\mathscr{J}$-trivial monoids, Semigroup Forum 19 (1980) 107-110.

[25] H. Straubing, Finite semigroup varieties of the form $\mathbf{V} * \mathbf{D}$, J. Pure Appl. Algebra 36 (1985) 53-94.

[26] H. Straubing and D. Thérien, Partially ordered finite monoids and a theorem of I. Simon, J. Algebra 119 (1988) 393-399.

[27] D. Thérien, Classification of finite monoids: the language approach, Theor. Comp. Sci. 14 (1981) 195-208.

[28] D. Thérien, Subword counting and nilpotent groups, in L. J. Cummings (ed.), Combinatorics on Words, Progress and Perspectives, Academic Press, 1983, 297-305.

[29] M. V. Volkov, On a class of semigroup pseudovarieties without finite pseudoidentity basis, Int. J. Algebra and Computation 5 (1995) 127-135.

[30] M. V. Volkov and I. A. Goldberg, Identities of semigroups of triangular matrices over finite fields, Mat. Zametki 73 (2003) 502-510 [Russian; Engl. translation Math. Notes 73 (2003) 474-481].

\section{J. Almeida \\ Departamento \\ de Matemática Pura \\ Faculdade de Ciências \\ Universidade do Porto \\ 4169-007 Porto \\ PORTUGAL \\ jalmeida@fc.up.pt}

S. W. Margolis

Department

of Mathematics

Bar Ilan University 52900 Ramat Gan ISRAEL

margolis@math.biu.ac.il
M. V. Volkov

Department

of Mathematics

and Mechanics

Ural State University 620083 Ekaterinburg

RUSSIA

Mikhail.Volkov@usu.ru 